\title{
Evaluation of Multiple Melting Peaks of Propylene-Ethylene Copolymers
}

\author{
Yuding FenG, ${ }^{\dagger, \dagger}$ Xiaorong JIN, and James N. HAY \\ School of Metallurgy and Materials, The University of Birmingham, \\ Edgbaston, Birmingham B15 2TT, U.K. \\ (Received July 16, 1997)
}

\begin{abstract}
Multiple melting peaks phenomena have been investigated in terms of compositional heterogeneity, crystallization temperature, cooling rate, heating rate, annealing and crystal structure for low ethylene content block and random propylene-ethylene copolymers. The experimental results suggested that the compositional heterogeneity and crystal morphology play an important role in the cause of multiple melting behavior.

KEY WORDS Multiple Melting Peaks / Propylene-Ethylene Copolymers / Recrystallization / Compositional Heterogeneity /
\end{abstract}

The melting behavior of isotactic polypropylene (i-PP) crystallized from the melt has been of interest since its initial synthesis ${ }^{1-3}$ because the melting temperature is largely affected by the chemical purity of the molecule. A common feature of all these investigations is the presence of multiple melting points attributed to several factors such as two different crystallographic forms, ${ }^{2}$ crystallites of varying degrees of perfection, ${ }^{3}$ difference in crystallite size ${ }^{4}$ etc. However, the origin of multiple melting points remains far from being completely understood.

i-PP is intrinsically brittle at low temperature and suffers from fracture problems in some applications. Products of greatly improved impact strength have been obtained by copolymerization of propylene with a small amount of ethylene using Ziegler-Natta catalysts. There are mainly two commercial propylene-ethylene copolymers in the market today, i.e., block and random copolymers. These copolymers prepared by heterogeneous catalysts exhibit compositional heterogeneity and it is generally accepted that heterogeneous catalysts have a plurality of active species. ${ }^{5,6}$ These copolymers contain a range of copolymers with different composition and sequence distribution. ${ }^{7}$

In the present work, multiple melting peaks behavior was studied on low ethylene content block and random propylene-ethylene copolymers.

\section{EXPERIMENTAL}

\section{Materials}

Commercially available polymers used in this study are listed in Table I.

Both bulk polymers were fractionated by temperature rising elution fractionation (TREF) technique. ${ }^{7}$ The results of compositional analysis for the fractions are shown in Tables II and III.

\section{Apparatus and Experimental Procedure}

A Perkin-Elmer differential scanning calorimeter, DSC-2, interfaced to a BBC-Master computer via an analogue to digital converter, was used to characterize the fractions. The temperature scale of the DSC was calibrated from the melting points of zone refined stearic acid ( $\mathrm{mp} 343.50 \mathrm{~K})$ and high purity metals such as indium $(\mathrm{mp} 429.78 \mathrm{~K})$, tin $(\mathrm{mp} 505.06 \mathrm{~K})$, lead $(\mathrm{mp} 600.50 \mathrm{~K})$, and zinc (mp $692.65 \mathrm{~K}$ ). The thermal response of the calorimeter was calibrated from the heat of fusion of ultra pure indium, $28.4 \mathrm{~J} \mathrm{~g}^{-1}$. Samples were encased in aluminum pans and a vacant aluminum pan was used as a reference.

A preparative TREF system was used to fractionate the copolymers. It consists of a double-wall fractionation column, pump compartments, and an oil filled thermostat. The fractionation column, $5 \mathrm{~cm}$ in diameter and $1.40 \mathrm{~m}$ in length, was made of a large double walled glass condenser and packed with fine silica sand support onto which the copolymers were crystallized by slow cooling from a hot xylene solution. The copolymer was eluted with xylene at a controlled flow rate and at a progressively increasing temperatures. Separation of

Table I. Propylene-ethylene copolymers

\begin{tabular}{lcl}
\hline \multicolumn{1}{c}{ Name } & Grade No. & Manufacture \\
\hline Block copolymer & RV210 & SOLVAY \\
Random copolymer & KV202X6380 & SOLVAY \\
\hline
\end{tabular}

Table II. The sequence distribution of block copolymer fractions

\begin{tabular}{lccccc}
\hline & \multicolumn{5}{c}{ Elution temperature $/{ }^{\circ} \mathrm{C}$} \\
\cline { 2 - 6 } Sequence & 105 & 107 & 110 & 115 & 120 \\
\hline E & 0.334 & 0.127 & 0.108 & 0.079 & 0.029 \\
P & 0.666 & 0.873 & 0.892 & 0.921 & 0.971 \\
PP & 0.574 & 0.857 & 0.862 & 0.904 & 0.950 \\
EP & 0.184 & 0.032 & 0.060 & 0.034 & 0.041 \\
EE & 0.334 & 0.111 & 0.078 & 0.062 & $\sim 0$ \\
PPP & 0.569 & 0.855 & 0.818 & 0.879 & 0.904 \\
PPE & 0.043 & 0.009 & 0.028 & 0.021 & 0.041 \\
EPE & 0.082 & 0.009 & 0.047 & 0.021 & 0.026 \\
PEP & 0.055 & 0.008 & 0.009 & 0.011 & 0.012 \\
EEP & 0.074 & 0.016 & 0.043 & 0.011 & 0.016 \\
EEE & 0.205 & 0.103 & 0.056 & 0.056 & $\sim 0$ \\
\hline
\end{tabular}

\footnotetext{
$\dagger$ To whom all correspondence should be addressed.

${ }^{\dagger \dagger}$ Present address: Department of Chemistry, University of Waterloo, Waterloo, Ontario, Canada N2L $3 G 1$.
} 
Table III. The sequence distribution of random copolymer fractions

\begin{tabular}{|c|c|c|c|c|c|}
\hline \multirow{2}{*}{ Sequence } & \multicolumn{5}{|c|}{ Elution temperature $/{ }^{\circ} \mathrm{C}$} \\
\hline & 90 & 95 & 100 & 105 & 110 \\
\hline $\mathrm{E}$ & 0.199 & 0.220 & 0.072 & 0.046 & 0.039 \\
\hline $\mathbf{P}$ & 0.801 & 0.780 & 0.928 & 0.954 & 0.961 \\
\hline PP & 0.668 & 0.618 & 0.882 & 0.924 & 0.925 \\
\hline EP & 0.265 & 0.318 & 0.091 & 0.059 & 0.065 \\
\hline $\mathrm{EE}$ & 0.066 & 0.064 & 0.027 & 0.016 & 0.010 \\
\hline PPP & 0.580 & 0.600 & 0.879 & 0.852 & 0.892 \\
\hline PPE & 0.167 & 0.103 & 0.026 & 0.069 & 0.061 \\
\hline EPE & 0.056 & 0.077 & 0.023 & 0.033 & 0.007 \\
\hline PEP & 0.066 & 0.096 & 0.037 & 0.027 & 0.020 \\
\hline EEP & 0.133 & 0.127 & 0.017 & 0.005 & 0.033 \\
\hline EEE & $\sim 0$ & $\sim 0$ & 0.018 & 0.014 & $\sim 0$ \\
\hline
\end{tabular}

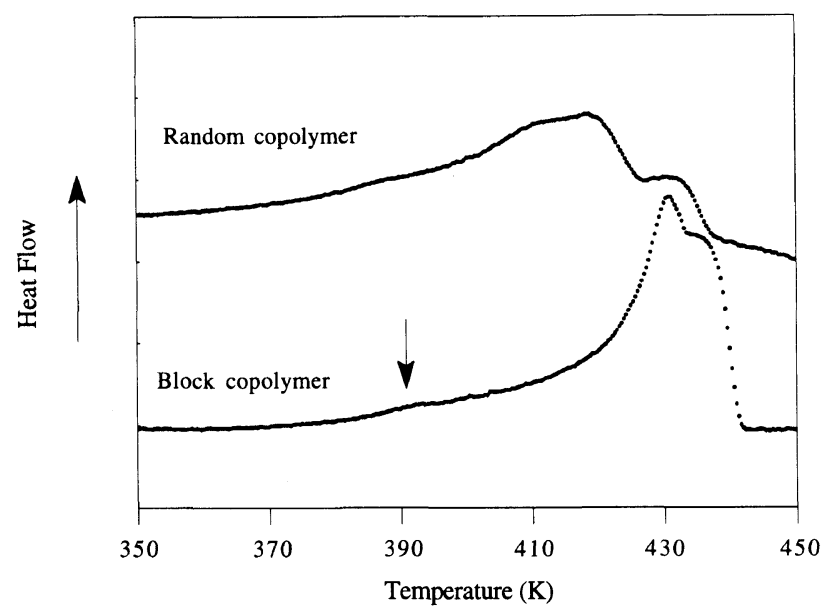

Figure 1. Melting curves of block and random propylene-ethylene copolymers.

molecular species was achieved by the temperature program. The fractions were collected at each elution temperature range, precipitated with methanol, filtered, dried in a vacuum oven at $60^{\circ} \mathrm{C}$, weighed and analyzed for compositional variations.

\section{RESULTS AND DISCUSSION}

Figure 1 shows the DSC melting endotherms of the block and random bulk proplyene-ethylene copolymers. Basically, for both block and random copolymers, multiple melting peaks can be observed between 400 to $430 \mathrm{~K}$. For block copolymer there is an additional small melting peak at about $390 \mathrm{~K}$. Both of the two copolymers are made by heterogeneous Ziegler-Natta catalyst, which often exhibit compositional heterogeneity. According to our previous compositional analysis, ${ }^{7}$ the block copolymer contains long ethylene sequences which are long enough to crystallize, while the random copolymer has no long ethylene sequences. So in DSC melting curve, the melting of polyethylene (PE) crystal can be observed at about $390 \mathrm{~K}$ for block copolymer, while no such melting peak for random copolymer. The following we discuss will be focused on the multiple melting peaks between 400 to $430 \mathrm{~K}$, which are attributed to the melt of PP crystals.

TREF fractionation was carried out for block and

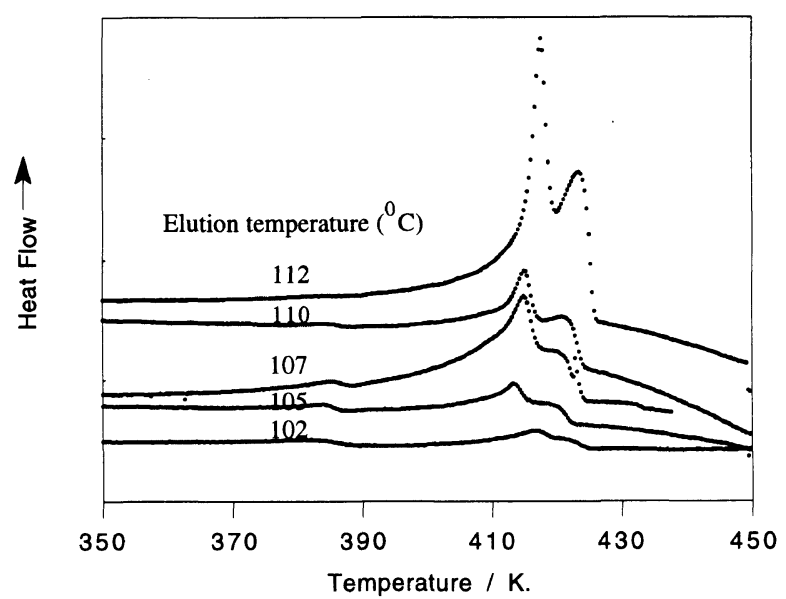

Figure 2a. DSC melting curves of block copolymer fractions $\left(10 \mathrm{~K} \mathrm{~min}^{-1}\right)$.

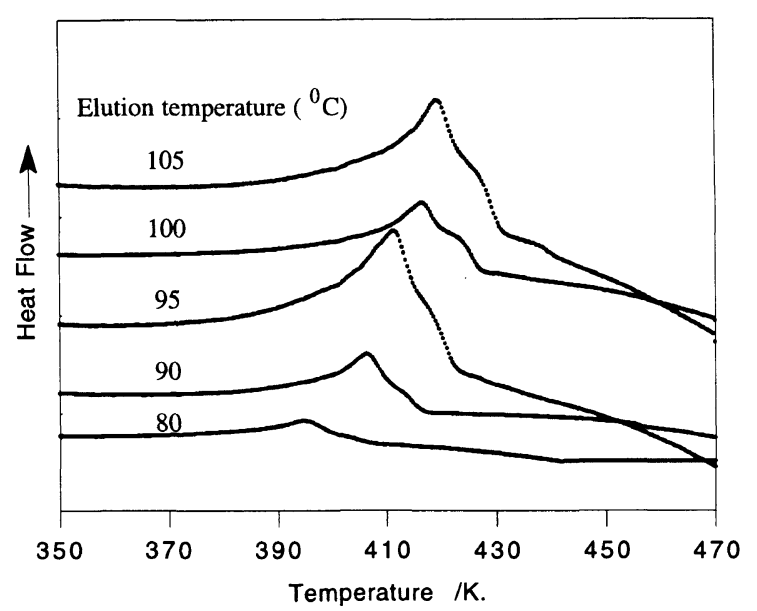

Figure 2b. DSC melting curves of the random fractions $\left(10 \mathrm{~K} \mathrm{~min}^{-1}\right)$.

random copoplymers. Figures 2(a) and 2(b) show the melting curves of fractions for the two copolymers. On eluting from 102 to $110^{\circ} \mathrm{C}$, low temperature melting peak $(390 \mathrm{~K})$ can be observed for the fractions of block copolymer. With increasing eluting temperature above $110^{\circ} \mathrm{C}$, the low temperature peak attributed to the melting of PE crystals becomes smaller and smaller, and finally disappeared. For random copolymer fractions, the melting peak shifted to higher temperature with increasing eluting temperature, resulting from the reduction of ethylene comonomer content in propylene chain.

All fractions show multiple melting peaks between 400 to $430 \mathrm{~K}$, attributed to the melting of PP crystal. Inspired by the TREF procedure, about $15 \mathrm{mg}$ of bulk random copolymer was melted at $470 \mathrm{~K}$ for $10 \mathrm{~min}$. Then the sample was successively crystallized at decreasing temperatures. The crystallization temperatures were 390 , $388,385,382,380,378,375,372,370,368,365 \mathrm{~K}$. The time for each crystallization step was $240 \mathrm{~min}$. The samples were finally cooled to $320 \mathrm{~K}$, heated at $5 \mathrm{~K} \mathrm{~min}^{-1}$ to $470 \mathrm{~K}$ and the melting endotherm and enthalpy recorded.

The DSC thermogram obtained by the method is presented in Figure 3. As can be seen, when using the staged cooling procedure, several well resolved melting 


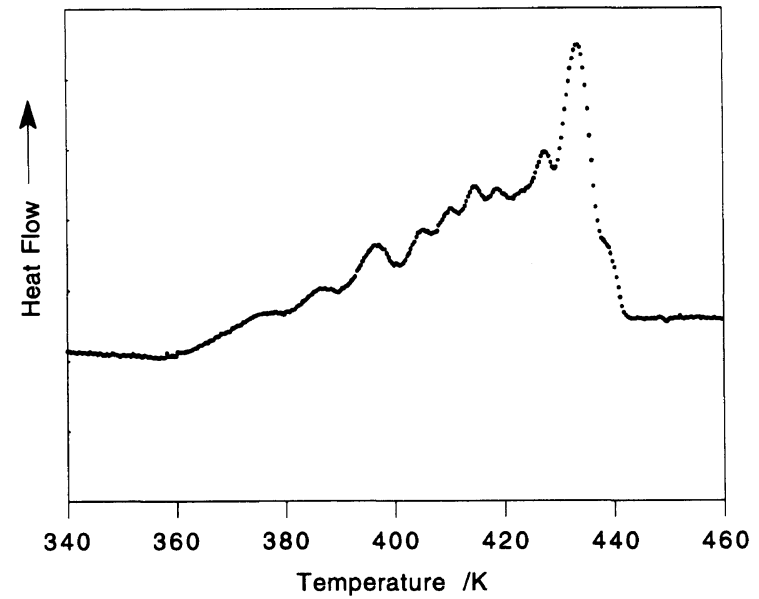

Figure 3. DSC melting curves of stepwise crystallized random PP.

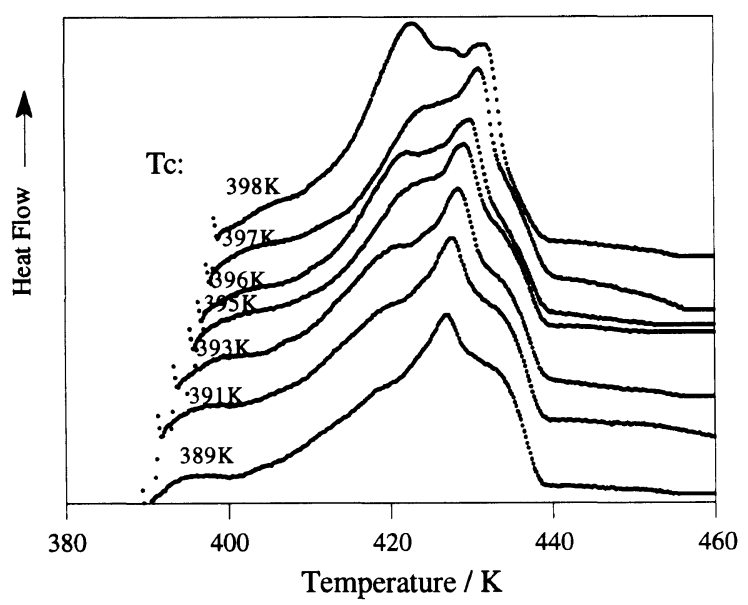

(a) Elution temperature, $107^{\circ} \mathrm{C}$

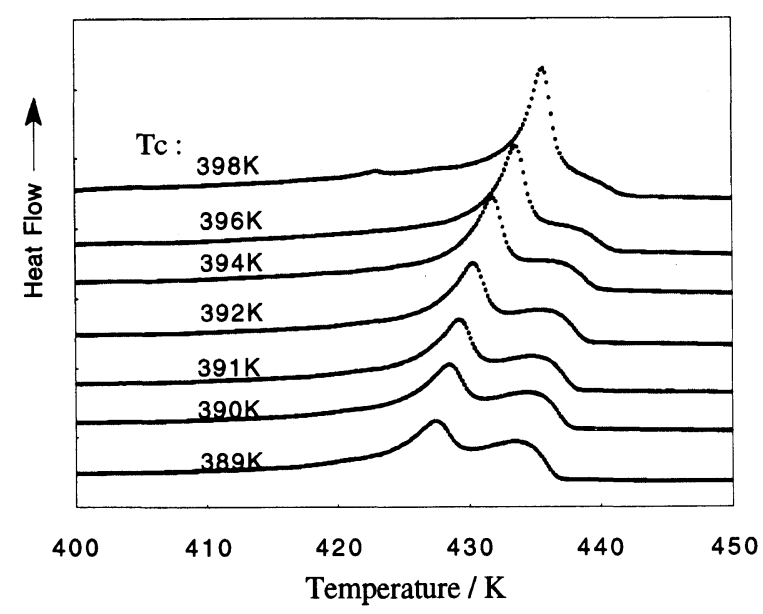

(b) Elution temperature, $112^{\circ} \mathrm{C}$

Figure 4. DSC curves for the melting of block PP fractions crystallized at various temperatures $\left(10 \mathrm{~K} \mathrm{~min}^{-1}\right)$.

endotherms are obtained during the heating cycle. These observations suggest separate crystallization of various types of copolymer crystallites during the staged cooling process. A similar observation has been obtained for the block copolymer. This phenomenon was tentatively interpreted as the formation, due to heterogeneity in comonomer unit distribution, of crystallites of different thickness and internal perfection giving rise to an in-

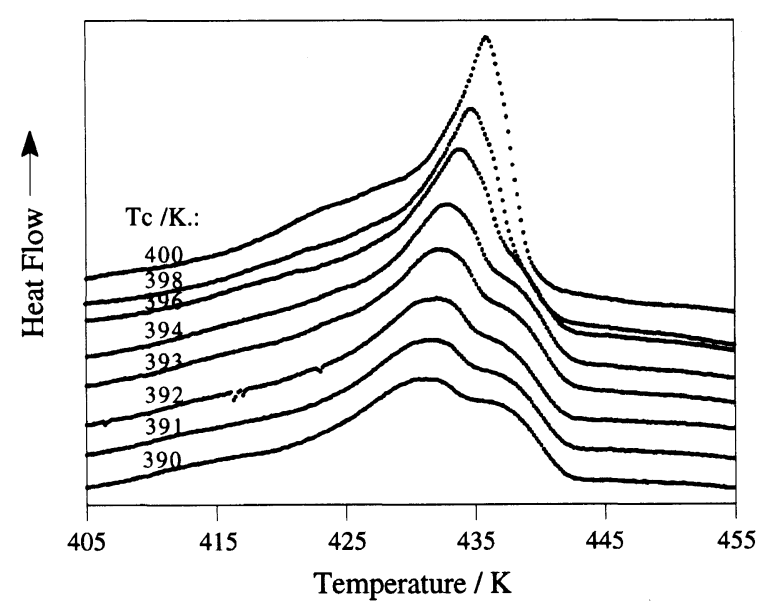

(c) Elution temperature, $115^{\circ} \mathrm{C}$

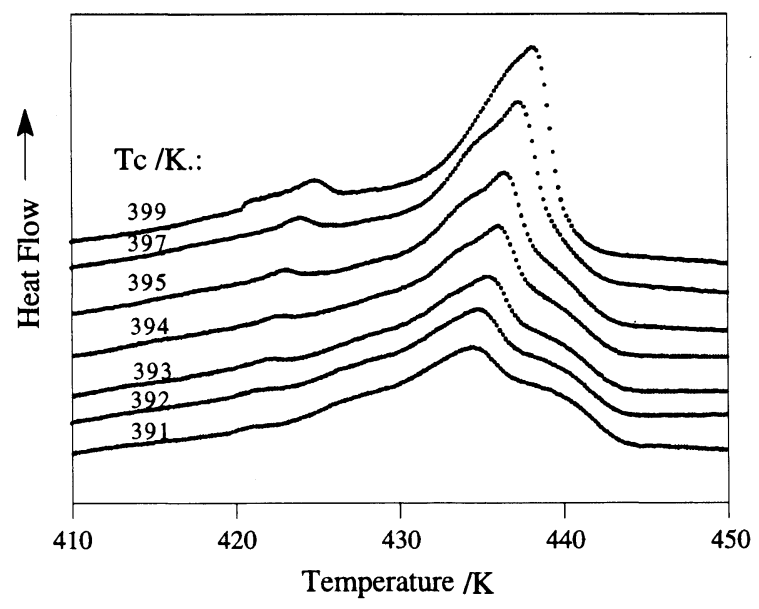

(d) Elution temperature, $120^{\circ} \mathrm{C}$

Figure 4. Melting curves of block PP fractions crystallized at different temperatures $\left(10 \mathrm{~K} \mathrm{~min}^{-1}\right)$.

homogeneous semicrystalline morphology.

Typical DSC curves for the melting of block and random copolymer fractions crystallized at different temperatures, are shown in Figures 4 and 5. A general feature of these curves is the appearance of multiple melting points, but details of the melting behavior are, however, dependent on the crystallization temperature. It should be expected that crystals grown under isothermal condition at high supercoolings are small and imperfect. They will melt at low temperatures. On isothermal crystallization, heating without prior cooling perfects these crystals continuously, and leads to higher melting species. However, as soon as these imperfect crystals are annealed, further perfection becomes increasingly difficult. So in this supercooling region, the multiple melting peaks observed are caused by perfection and/or recrystallization of imperfect crystals initially grown during isothermal crystallization. For sample eluted at different temperature, it can be seen that with decreasing of eluting temperature the melting curves become broader and more complex. This suggested that the less ethylene content in propylene chain, the perfect are the PP crystals formed.

On cooling at $2.5,5,10,20,40,80 \mathrm{~K} \mathrm{~min}^{-1}$ and subsequently heating at $10 \mathrm{~K} \mathrm{~min}^{-1}$, the resulting ther- 


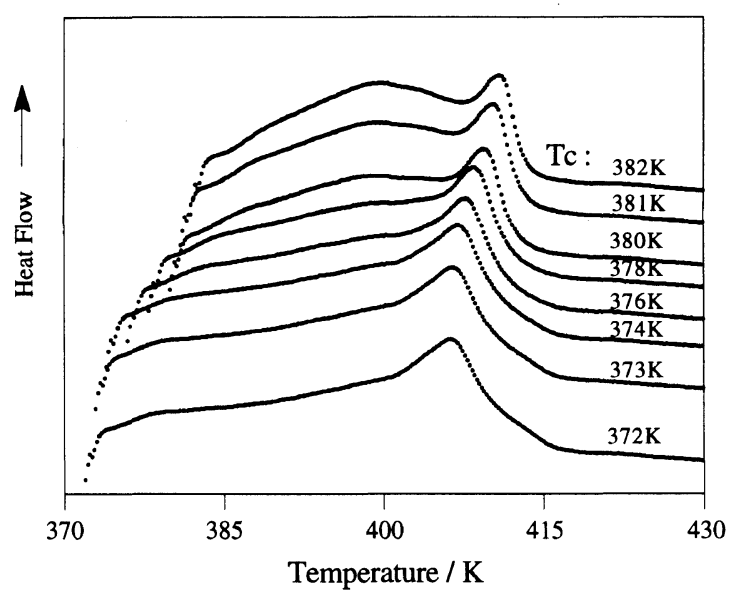

(a) Elution temperature, $90^{\circ} \mathrm{C}$

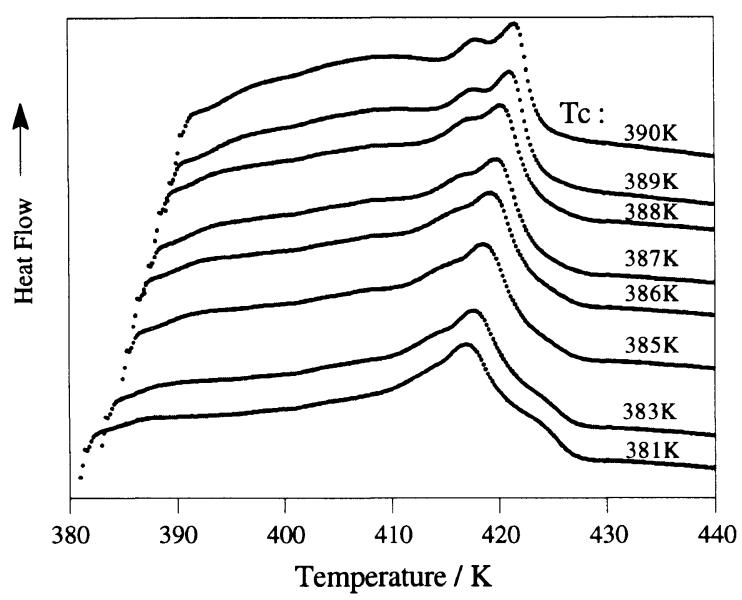

(b) Elution temperature, $100^{\circ} \mathrm{C}$

Figure 5. DSC curves for the melting of random PP fractions crystallized at different temperatures $\left(10 \mathrm{~K} \mathrm{~min}^{-1}\right)$.

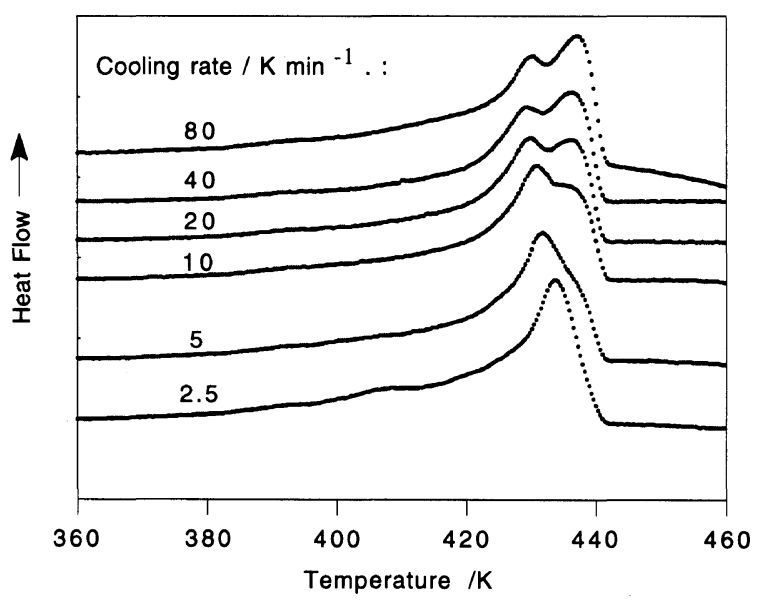

Figure 6. Effect of cooling rate on the melting curves of block PP $\left(10 \mathrm{~K} \mathrm{~min}^{-1}\right)$.

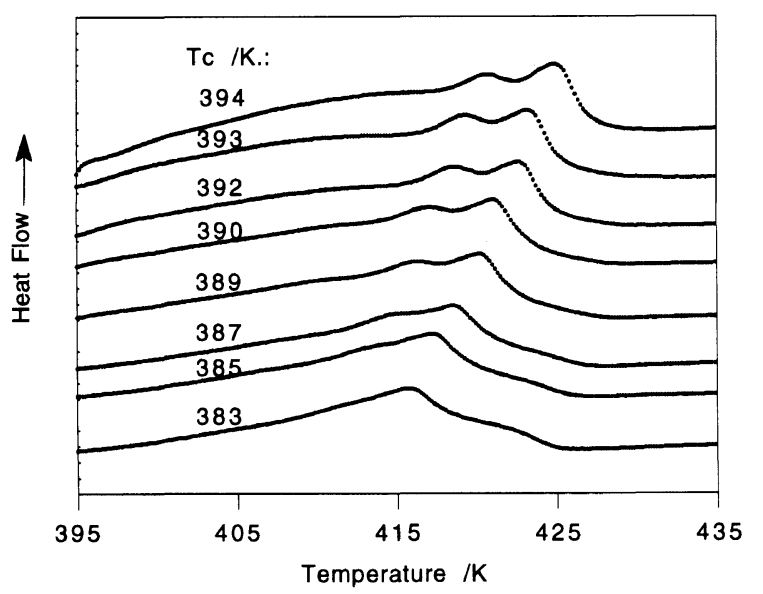

(c) Elution temperature, $105^{\circ} \mathrm{C}$

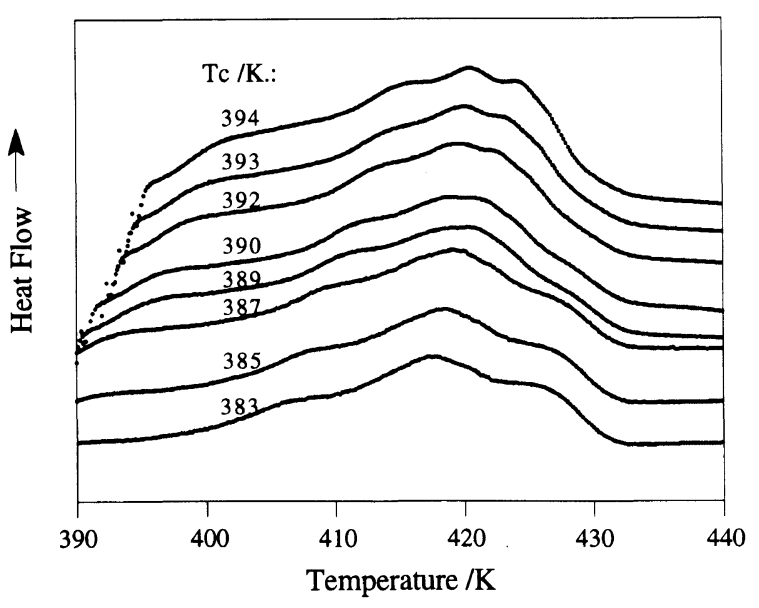

(d) Elution temperature, $110^{\circ} \mathrm{C}$

Figure 5. Melting curves of random PP fractions crystallized at different temperatures $\left(10 \mathrm{~K} \mathrm{~min}^{-1}\right)$.

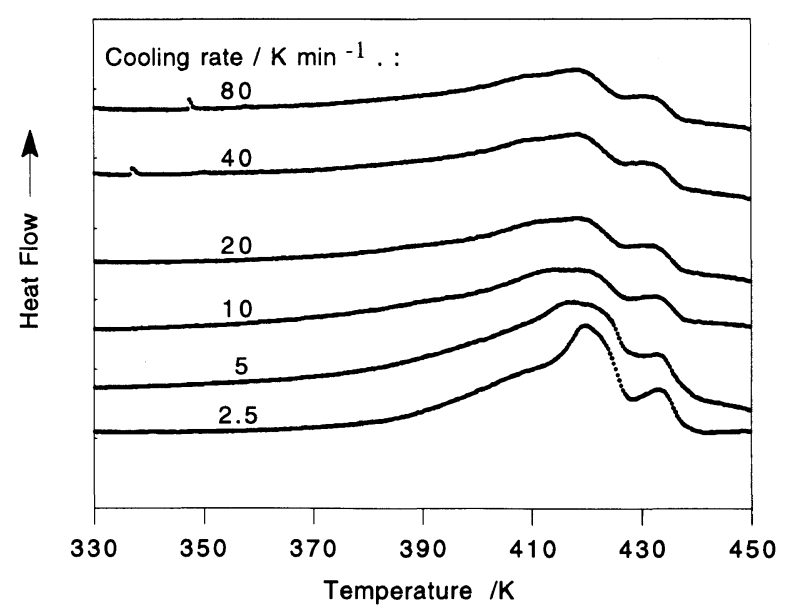

Figure 7. Effect of cooling rate on the melting curves of random PP $\left(10 \mathrm{~K} \mathrm{~min}^{-1}\right)$. 


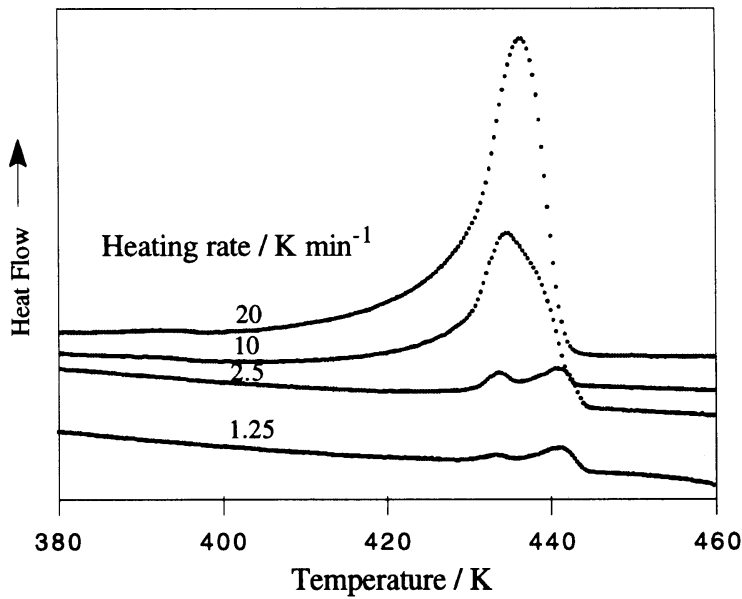

Figure 8. Effect of heating rate on the DSC melting curves of block copolymer.

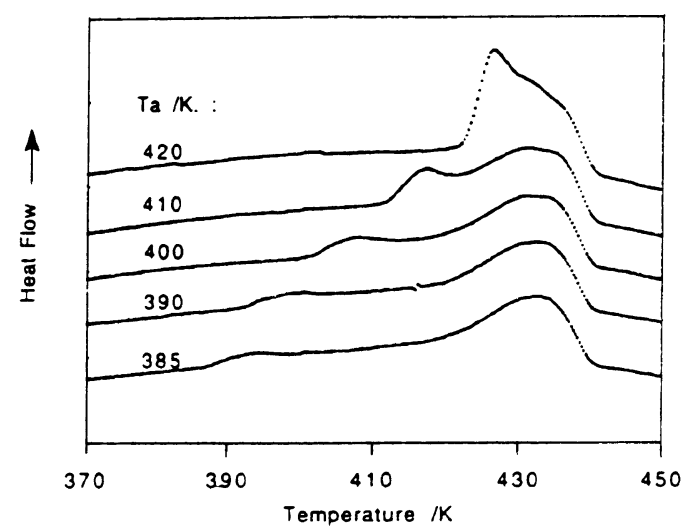

Figure 9. Effect of annealing temperature on the melting curves of a block copolymer fraction eluted at $117^{\circ} \mathrm{C}$ (annealed $45 \mathrm{~min}$ ).

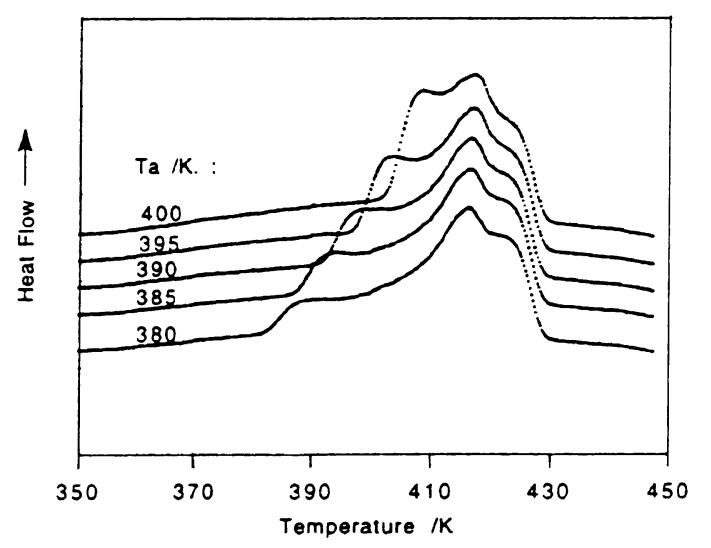

Figure 10. Effect of annealing temperature on the melting curves of a random copolymer fraction eluted at $105^{\circ} \mathrm{C}$ (annealed $45 \mathrm{~min}$ ).

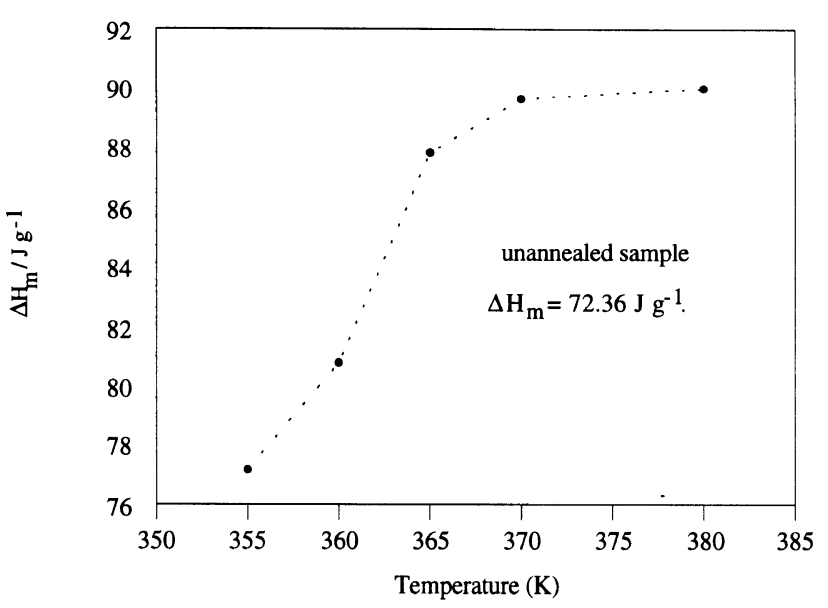

(a) Random copolymer

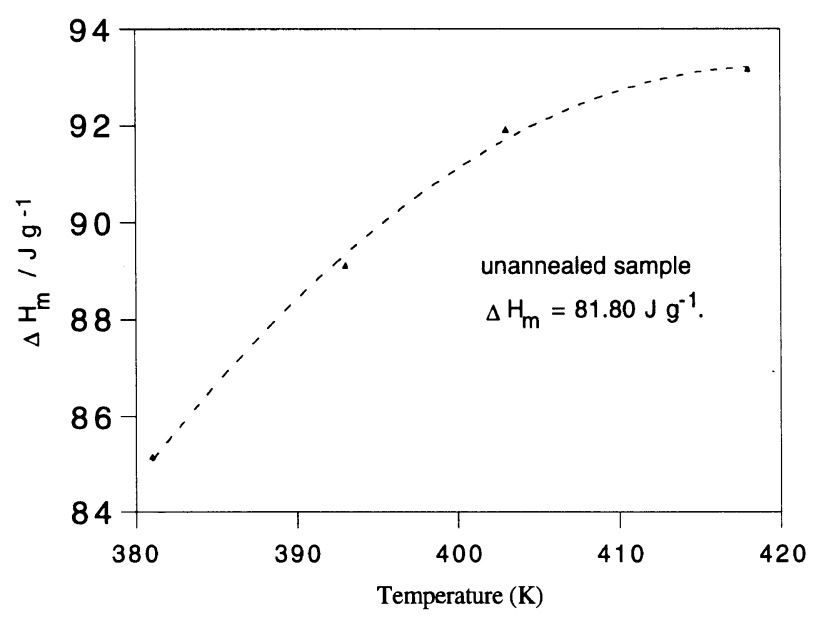

(b) Block copolymer

Figure 11. Relationship between the heat of fusion and annealing temperature.

mograms for block and random copolymers are shown in Figures 6 and 7. It is to be expected that, when the molten copolymer was rapidly cooled in the DSC, defects in the crystalline zones occur which on reheating the melt at a lower temperature. After melting, it was followed by recrystallization and further remelting while the DSC scan was in progress. On the other hand, if the cooling rate is sufficiently slow, the polymer chain can crystallize better and much less defects exist in crystalline region, so the melting curve only gives a single peak.

For samples crystallized under the same conditions, the melting behavior depended strongly on the heating rate, as case be seen in Figure 8 for the block copolymer. Recrystallization involves crystal perfection and crystal thickening. These crystals melt at higher temperatures depending upon the degree of perfection achieved. On increasing the heating rate, less time is available for the crystal perfection to take place. And some fractions of the original crystals formed can melt and recrystallise at higher temperatures leading to thicker and more perfect crystals and these would also melt at higher temperature. As the heating rate is increased, less and less time is available for recrystallization to take place and hence the fraction of the crystallized fraction undergoing this 


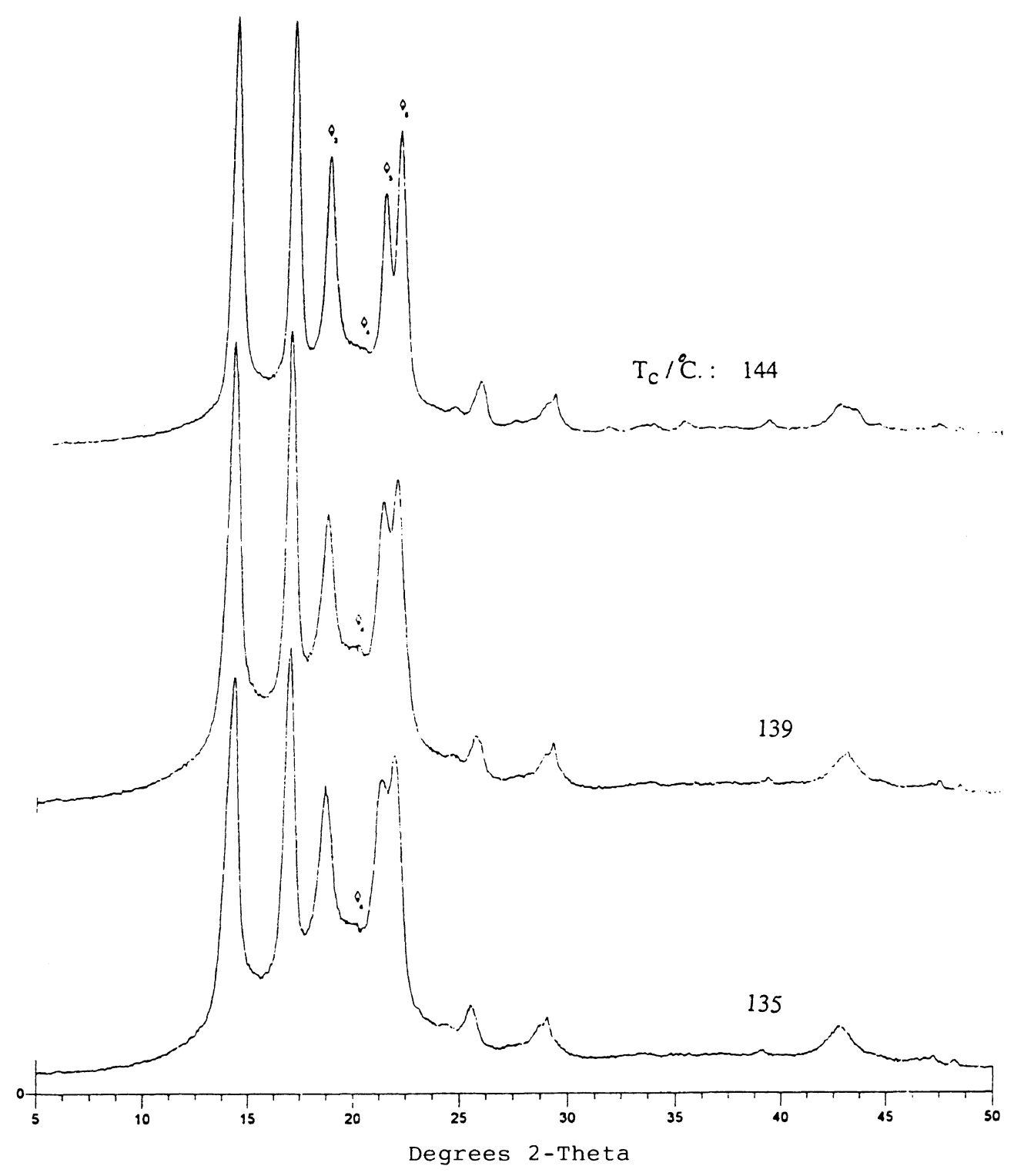

Figure 12. Effect of crystallization temperature on X-ray diffraction patterns for block copolymer.

process decreases. Thus at a very high heating rate, no reorganization takes place and hence only one melting peak is observed. On the other hand, if the heating rate is extremely low such that most of the isothermally crystallized fractions is reorganized or recrystallized, then also a single peak is observed.

The multiple melting behavior has also been observed for annealed block and random fractions. The DSC thermographs of a block copolymer fraction (eluted at $117^{\circ} \mathrm{C}$ ) and a random copolymer fraction (eluted at $105^{\circ} \mathrm{C}$ ) annealed at different temperatures are shown in Figures 9 and 10. It can be seen that a shoulder peak is present at lower temperature as well as the main melting transition. As the annealing temperature is increased this shoulder shifts upwards and joins the main peak. The position of the main melting peak does not change. At an annealing temperature of $420 \mathrm{~K}$, both peaks merge to a single melting peak. This clearly demonstrates the presence of recrystallization during the annealing process. From the relationship between the heat of fusion and annealing temperature (Figure 11), it can be seen that the crystallinity increased with the annealing temperature.

Some authors ${ }^{2,8}$ have suggested that the lower melting peaks are attributable to the $\gamma$-form of polypropylene with the lowest endotherm melting and recrystallizing during a scan to a more ordered $\gamma$-phase. If this were the case, then the $\gamma$-phase would show predominantly in the X-ray diffraction. Figure 12 showed the WAXD diffractogram of bulk block copolymer crystallized at different temperature. It showed that the $\alpha$-form is predominant. Figure 13 showed the WAXD diffractogram of the block copolymer crystallized rapidly (cooled in air) and slowly (cooled on hot-press). It also showed that the $\alpha$-form is dominant.

So it can be concluded that the multiple melting peaks of low ethylene content propylene-ethylene copolymers are resulted from the compositional heterogeneity of samples and the crystal morphology. 


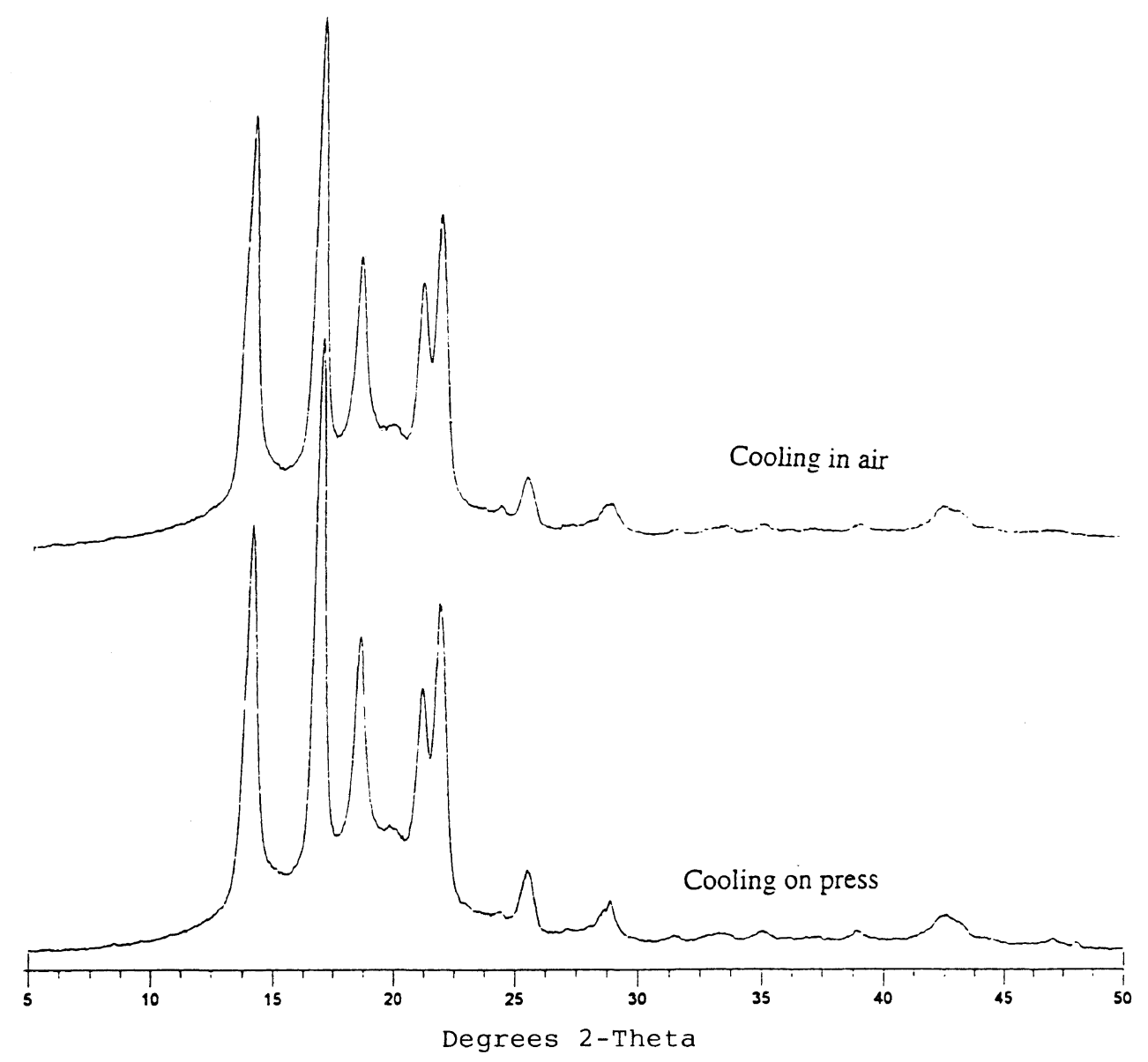

Figure 13. Effect of thermal treatment on $\mathrm{X}$-ray diffraction patterns for block copolymer.

\section{CONCLUSION}

Multiple melting point phenomena have been discussed in terms of compositional heterogeneity, crystallization temperature, cooling rate, heating rate, annealing and crystal structure for low ethylene content propylene-ethylene copolymers. TREF fractionation was carried out to investigate the effect of compositional heterogeneity on the melting behavior. The experimental results suggested that the perfection and recrystallization play an important role in the cause of multiple melting behavior.

\section{REFERENCES}

1. G. Natta, G. Mazzanti, G. Crespi, and G. Moraglio, Chim. Ind., 39, 275 (1957)

2. J. Kardos, A. W. Christiansen, and E. Baer, J. Polym. Sci., Part A-2, 4, 477 (1966).

3. K. D. Pae and J. A. Sauer, J. Polym. Sci., 12, 1901 (1968).

4. R. J. Samuels, J. Polym. Sci., Polym. Phys. Ed., 13, 1417 (1975).

5. C. Cozewith, Macromolecules, 20, 1237 (1987).

6. T. Hayashi, Y. Inoue, and R. Chujo, Macromolecules, 21, 3139 (1988).

7. Y. Feng, X. Jin and J. N. Hay, J. Polym. Sci., Polym. Phys. Ed., in press.

8. Y. Fujiwara, Colloid Polym. Sci., 253, 273 (1975). 